\title{
Why Instantaneous Values of the "Covariant" Lyapunov Exponents Depend upon the Chosen State-Space Scale
}

\author{
Wm.G. Hoover, C.G. Hoover \\ Ruby Valley Research Institute \\ Highway Contract 60, Box 601 \\ Ruby Valley, Nevada 89833 \\ E-mail: hooverwilliam@yahoo.com
}

Received: 20 November 2013; accepted: 9 December 2013; published online: 31 December 2013

\begin{abstract}
We explore a simple example of a chaotic thermostated harmonic-oscillator system which exhibits qualitatively different local Lyapunov exponents for simple scale-model constant-volume transformations of its coordinate $q$ and momentum $p:\{q, p\} \rightarrow\{(Q / s),(s P)\}$. The time-dependent thermostat variable $\zeta(t)$ is unchanged by such scaling. The original $(q p \zeta)$ motion and the scale-model $(Q P \zeta)$ version of the motion are physically identical. But both the local Gram-Schmidt Lyapunov exponents and the related local "covariant" exponents change with the change of scale. Thus this model furnishes a clearcut chaotic time-reversible example showing how and why both the local Lyapunov exponents and covariant exponents vary with the scale factor $s$.
\end{abstract}

Key words: Lyapunov Instability, Thermostats, Chaotic Dynamics

\section{LOCAL AND GLOBAL GRAM-SCHMIDT COVARIANT VECTORS AND EXPONENTS}

The popularity of the time-dependent (or "local", or "instantaneous") covariant Lyapunov vectors and their associated exponents as descriptions of chaotic motion seems to us to be linked to a (false) impression extracted from the literature. Some of the literature implies that these descriptors have a special significance independent of such details as the coordinate system used to describe them. A selected literature, some of it quite clear, can be found in References 1-7. If the chosen coordinate system were really insignificant it would be hard to understand a simple, but nonchaotic, counterexample: the one-dimensional harmonic oscillator, which exhibits a strong dependence of its largest local Lyapunov exponent $\lambda_{1}(t)$ on the chosen Cartesian coordinate system $[1,8,9]$.

We remind the reader that this local instantaneous Lyapunov exponent $\lambda_{1}(t)$ (the largest of them when time averaged) measures the local rate of divergence of two nearby trajectories. Think of them as a reference trajectory and a satellite trajectory, with the satellite constrained to remain near the reference. It is unnecessary to consider exponents beyond the first to understand why it is that the local Lyapunov exponents, covariant or not, are in fact not scale-independent and do indeed depend upon the chosen coordinate system or set of measurement units. The oftrepeated statement that the local covariant exponents are "norm-independent" should not be misunderstood (as we did) to mean that the exponents are independent of a scale factor, as in a change of units from cgs to MKS.

Here we focus on a simple chaotic continuous-flow example [10], the thermostated three-dimensional flow of a harmonic oscillator with coordinate $q$, momentum $p$, and friction coefficient $\zeta(t)$ in the unscaled $(q, p, \zeta)$ phase space:

$\dot{q}=p ; \dot{p}=-q-\zeta p ; \dot{\zeta}=p^{2}-T(q) ; T(q)=1+\epsilon \tanh (q)$.

The variation of temperature with coordinate $T(q)$ makes possible dissipation, and phase-volume shrinkage, $\dot{\otimes}<0$, onto a torus, or a strange attractor with fractional dimensionality, or a one-dimensional limit cycle. For the evolution of this model see References 11-14. 


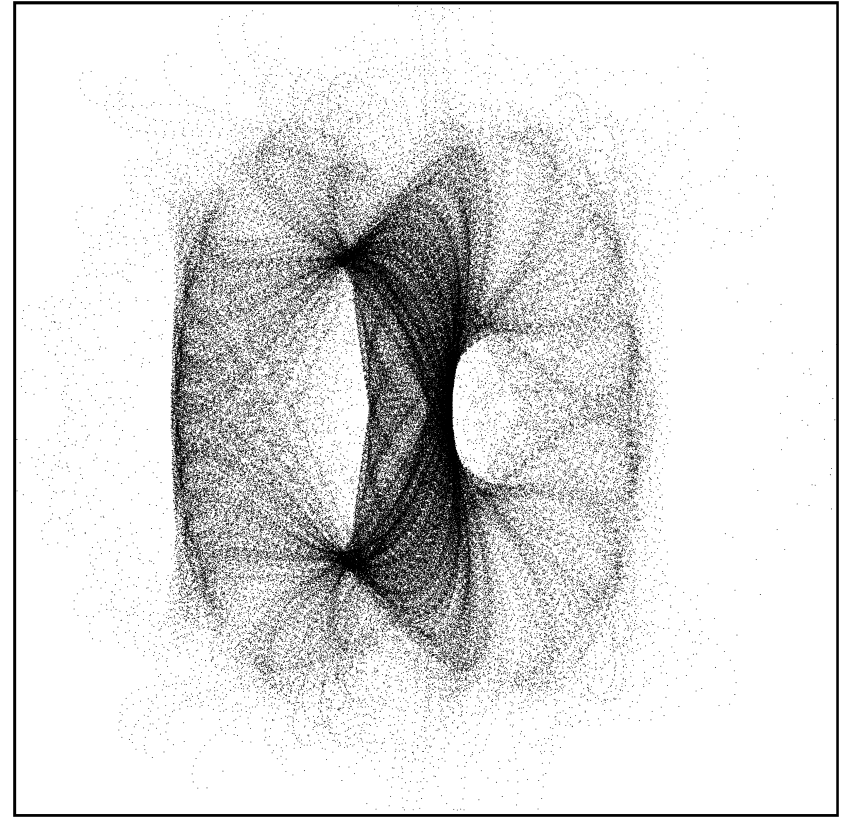

Fig. 1. Chaotic attractor $\zeta(q)$ projection for $\epsilon=0.20$ with fourthorder Runge-Kutta timestep $d t=0.001$ using 200, 000 equallyspaced points from the last half of a 40,000, 000 timestep simulation. The abscissa and ordinate scales range from -4.0 to +4.0

For simplicity's sake the oscillator mass and force constant, as well as Boltzmann's constant, are all chosen equal to unity here. For $\epsilon=0.20$ and with initial values $(q=$ $0, p=5, \zeta=0)$ the motion generates a chaotic strange attractor, with two time-averaged nonzero Lyapunov exponents $\lambda_{1} \simeq+0.01 ; \lambda_{3} \simeq-0.01$ and with a time-averaged rate of phase-volume contraction imposed by the friction coefficient $\zeta$,

$$
\begin{gathered}
\langle\dot{\otimes} / \otimes\rangle=\langle(\partial \dot{q} / \partial q)+(\partial \dot{p} / \partial p)+(\partial \dot{\zeta} / \partial \zeta)\rangle= \\
=0-\langle\zeta\rangle+0=\lambda_{1}+\lambda_{2}+\lambda_{3} \simeq-0.0003 .
\end{gathered}
$$

Regular, limit-cycle, and chaotic solutions can all be found by following the related work carried out in Reference 10. These solutions' details depend upon the initial conditions as well as the value of the maximum temperature gradient $\epsilon \equiv(d T / d q)_{q=0}$.

Figures 1 and 2 show both a typical chaotic strange attractor (positive $\lambda_{1}$, generated with $\epsilon=0.20$ ) and an unusually elaborate limit cycle (zero $\lambda_{1}$, generated with $\epsilon=0.37$ ). The time required for the appearance of such limit cycles can be hundreds of millions, or even billions, of timesteps. Although fourth-order Runge-Kutta timesteps ranging from 0.0005 to 0.05 produce such a cycle a careful look at Figure 2 reveals a disconcerting dependence of cycle topology on the time step(!).

The "local" time-dependent value of the largest Lyapunov exponent $\lambda_{1}(t)$ describes the rate at which two nearby $(q, p, \zeta)$ trajectories tend to separate:

$$
\lambda_{1}(t) \equiv(d \ln r / d t) ; r \equiv \sqrt{\delta q^{2}+\delta p^{2}+\delta \zeta^{2}} \simeq e^{+\lambda_{1} t} .
$$

In the simple Gram-Schmidt picture (and unlike the covariant picture with its nonorthogonal, but still normalized vectors) adding in the second Lyapunov exponent $\lambda_{2}$ gives the rate of divergence of the area defined by three nearby trajectories (the reference and two satellites), $\propto$ $\exp \left[+\lambda_{1} t+\lambda_{2} t\right]$. The third Gram-Schmidt exponent is needed to describe the divergence (or shrinkage) rate associated with the volume associated with four nearby trajectories, $\propto \exp \left[+\lambda_{1} t+\lambda_{2} t+\lambda_{3} t\right]$. In these three Gram-Schmidt definitions the time $t$ is understood to be sufficiently long for convergence of the exponents.

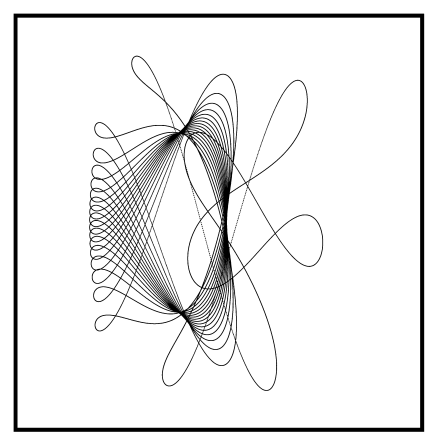

$\mathrm{dt}=\mathbf{0 . 0 5}$

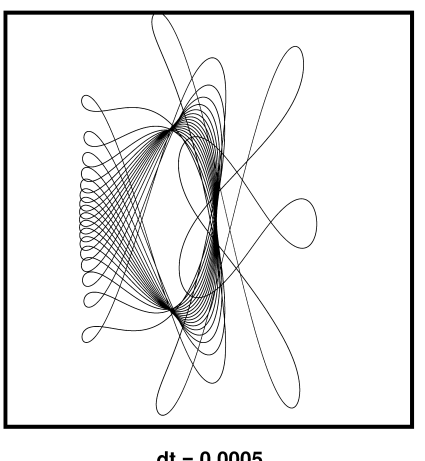

$\mathrm{dt}=\mathbf{0 . 0 0 0 5}$
Fig. 2. Limit cycles' $\zeta(q)$ projections for $\epsilon=0.37$ using timesteps of 0.05 and 0.0005 . The abscissa and ordinate range from -4.0 to +4.0 . For a particularly dramatic illustration of timestepdependent trajectory topology the brave reader is encouraged to compare the limit cycles for timesteps of $0.0199,0.0200$, and 0.0201 using the classic fourth-order Runge-Kutta integrator. Begin with $(q, p, \zeta)=(0,5,0)$ and discard the first 500,000,000 timesteps to follow this suggestion up.

Typically, these time-averaged exponents do not depend on the coordinate system used to describe the system because the divergence is exponential, and so depends only on the units of time, not those of space or momentum. Two identical chaotic systems, one described with MKS units and the other with cgs units exhibit the same (time-averaged) rates of divergence even though the mass and length scales differ. It is also possible, usual, and useful to define "local" or "instantaneous" Lyapunov exponents by following two or more constrained trajectories and measuring their tendencies to separate or approach each other as a function of the time of measurement $[1-9,15]$. The MKS and cgs values of these local exponents differ. The Gram-Schmidt Lyapunov exponents are simply the time averages of these instantaneous values:

$$
\lambda_{1}=\left\langle\lambda_{1}(t)\right\rangle ; \lambda_{2}=\left\langle\lambda_{2}(t)\right\rangle ; \lambda_{3}=\left\langle\lambda_{3}(t)\right\rangle \ldots .
$$

In typical situations, time-reversible and phase-volumeconserving Hamiltonian systems have "paired" GramSchmidt exponents, with the instantaneous identities:

$$
\lambda_{1}(t)+\lambda_{N}(t)=\lambda_{2}(t)+\lambda_{N-1}(t)=\cdots \equiv 0 .
$$


But in exceptional cases (like the collision of two many-body chunks of solid) [15] this pairing can be violated. Because the pairing reflects the time-reversibility of the Hamiltonian equations of motion this lost symmetry is simply a symptom that the "past" can be sufficiently different to the future. But because the time-averaged Hamiltonian exponents often exist in $\pm \lambda(t)$ pairs, the largest exponent in either time direction is typically equal to (the negative of) the smallest exponent in that same time direction. We reiterate that this symmetry can be violated, for short times, in response to inhomogeneities or to "external perturbations". [15]

By now many groups [1-6] have illustrated the algebraic steps necessary to map the "covariant" exponents from one coordinate system to another. A careless reader of some of this work might well conclude (as we did) that "covariant" vectors and exponents are somehow coordinate-frame independent. A careful reader will instead note that because reference trajectories and nearby satellite trajectories in one coordinate system can always be related to those in another, that the offset vectors linking pairs of trajectories are likewise simply related so that the (different) exponents in both frames can be computed.

It is not always emphasized that the exponents (even the largest, which is "covariant") themselves vary from frame to frame. For instance, in a useful and clarifying work, Posch [2,9] selected a spring-pendulum for his demonstration. His two chosen frames were Cartesian and polar coordinates. The constant-energy spring-pendulum dynamics can be described in either one of the three-dimensional subspaces of the four-dimensional spaces in which the motion is described, $\left(x, y, p_{x}, p_{y}\right)$ or $\left(r, \theta, p_{r}, p_{\theta}\right)$. Expressions linking the covariant exponents in these two frames (which are different) are given in his paper.

Here we consider again the $(q, p, \zeta)$ oscillator, a onedimensional rather than a two-dimensional system, and described in a three-dimensional phase space. The description can be carried out with $\{q, p, \zeta, \dot{q}, \dot{p}, \dot{\zeta}\}$ or with "scaled variables" $\{Q, P, \zeta, \dot{Q}, \dot{P}, \dot{\zeta}\}$, where the two sets of variables are related by the scaling $Q=2 q, P=(p / 2)$ :

$$
\begin{gathered}
\dot{q}=p ; \dot{p}=-q-\zeta p ; \dot{\zeta}=p^{2}-T(q) ; T(q)=1+\epsilon \tanh (q) . \\
\dot{Q}=4 P ; \dot{P}=-(Q / 4)-\zeta P ; \dot{\zeta}=4 P^{2}-T(Q) ; \\
T(Q)=1+\epsilon \tanh (Q / 2) .
\end{gathered}
$$

Because the temperature depends upon the coordinate [ so that $T$ varies from $(1-\epsilon)$ to $(1+\epsilon)]$, this model [10] is a generalized version of the Nosé-Hoover oscillator described in Reference 12. The two sets of equations generate trajectories which are identical if the coordinate and momentum axes are scaled because [ $Q=s q]$ and $[P=(p / s)]$. Here we compare $s=1$ and $s=2$. The friction coefficient $\zeta$, which directs the squared momentum toward the local kinetic temperature $T(q)$, is exactly the same function of time in both the original unscaled and the scaled coordinate systems. Thus the basic trajectories in $(q p \zeta)$ space and $(Q P \zeta)$ space are identical scale models of each other apart from factors of two in the directions associated with the length and momentum. Figure 3 shows the variation of the largest Lyapunov exponent with time along the relatively-simple limit cycle obtained when $\epsilon=0.50$. Notice that the local Lyapunov exponents $\lambda_{1}(t, q, p, \zeta, s)$ and $\lambda_{1}(t, Q, P, \zeta, s)$ are indeed sensitive to the scale factor $s$.

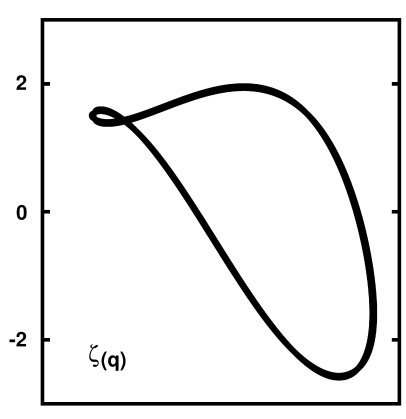

$-3.0<q=(Q / s)<0.5$

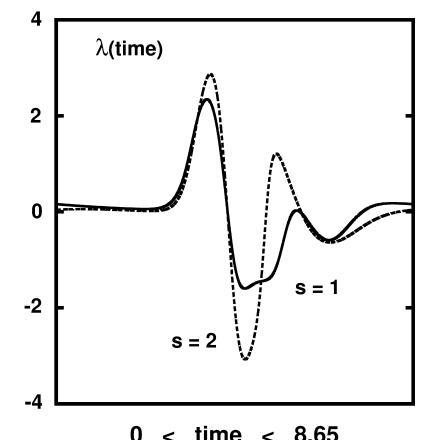

$0<$ time $<8.65$
Fig. 3. Local values of the largest Lyapunov exponents (right) for the limit cycle with $\epsilon=0.50$ (left). The time-averaged exponents are equal to 0.0

This computation shows that the local exponents are quite different. Why is that? Here it is because the stretch rates depend on the scale factor $s$. The rates of stretching of pairs of (infinitesimal) tangent-space "unit vectors" parallel to $q=\left(s^{-1} Q\right)$ or parallel to $p=\left(s^{+1} P\right)$ are different:

$$
\dot{\delta} q=s^{-1} \dot{\delta} Q ; \dot{\delta} p=s^{+1} \delta P
$$

so that the corresponding local Lyapunov exponents in these two hypothetical cases would also vary with $s$. We exhibit this example here to emphasize the point that even the local values of the Lyapunov exponents depend on the chosen coordinate system. The global exponents for Hamiltonian systems do not show this dependence. In an email of 18 September 2013 Harald Posch showed that the global exponents for a doubly-thermostated oscillator do depend on the scale factor $s$ but not on the norm. Posch compared the exponents using both the usual $n=2$ norm and the unusual $n=3$ one:

$$
r^{n}=|d q|^{n}+|d p|^{n}+|d \zeta|^{n}+|d \xi|^{n} .
$$

\section{CONCLUSION}

Enthusiastic fans of the MKS system of units cannot agree with the ardent fans of the cgs system when it comes to the local exponents, either covariant or Gram-Schmidt. Disinterested observers will note that one set of results can be converted to the other, with the whole spectrum as well as its fluctuations dependent on the chosen coordinate system. The impression that "covariant" exponents are somehow uniquely special still seems to us specious despite their norm-independence. 


\section{ACKNOWLEDGMENTS}

We thank Roger Samelson for the stimulating emails which led us careless readers to our current understanding and reinvestigation of the dependence of the local covariant exponents on the chosen coordinate system. Likewise emails from Harald Posch were very useful. They pointed out that scaling transformations, such as the MKS-cgs distinction and the $s^{+1}$ and $s^{-1}$ combination, are not usually thought of as acceptable norms, although no calculation at all is possible without first choosing some phase space. We also appreciate our longstanding correspondences on this subject with Pavel Kuptsov (see also his arXiv contributions) and Franz Waldner as well as a useful remark by Günter Radons and are happy to confess that at last we "understand".

\section{References}

[1] H. Bosetti, H.A. Posch, Ch. Dellago and Wm.G. Hoover, Time-Reversal Symmetry and Covariant Lyapunov Vectors for Simple Particle Models in and out of Thermal Equilibrium, arXiv:1004.4473, Version 1 (2010); Physical Review E 82, 046218 (2010).

[2] H.A. Posch, Symmetry Properties of Orthogonal and Covariant Lyapunov Vectors and Their Exponents, arXiv:1107.4032 (2012); Journal of Physics A: Mathematical and Theoretical 46, 254006 (2013).

[3] H-L. Yang and G. Radons, Comparison between Covariant and Orthogonal Lyapunov Vectors, Physical Review E 82, 046204 (2010).
[4] F. Ginelli, P. Poggi, A. Turchi, H. Chaté, R. Livi, and A. Politi, Characterizing Dynamics with Covariant Lyapunov Vectors Physical Review Letters 99, 130601 (2007).

[5] M. Romero-Bastida, D. Pazó, J.M. López, and M.A. Rodríguez, Structure of Characteristic Lyapunov Vectors in Anharmonic Hamiltonian Lattices, Physical Review E 82, 036205 (2010).

[6] C.L. Wolfe and R.M. Samelson. An Efficient Method for Recovering Lyapunov Vectors from Singular Vectors, Tellus 59A, 355-366 (2007).

[7] W.G. Hoover and H.A. Posch, Direct Measurement of Lyapunov Exponents, Physics Letters A 113, 82-84 (1985).

[8] Wm.G. Hoover and C.G. Hoover, Local Gram-Schmidt and Covariant Lyapunov Vectors and Exponents for Three Harmonic Oscillator Problems, Communications in Nonlinear Science and Numerical Simulation 17, 1043-1054 (2012).

[9] Wm.G. Hoover, C.G. Hoover, and H.A. Posch, Lyapunov Instability of Pendulums, Chains, and Strings, Physical Review A 41, 2999-3004 (1990).

[10] H.A. Posch and Wm.G. Hoover, Time-Reversible Dissipative Attractors in Three and Four Phase-Space Dimensions, Physical Review E 55, 6803-6810 (1997).

[11] Wm.G. Hoover, Canonical Dynamics: Equilibrium PhaseSpace Distributions, Physical Review A 31, $1695-97$ (1985).

[12] H.A. Posch, Wm.G. Hoover, and F.J. Vesely, Canonical Dynamics of the Nosé Oscillator: Stability, Order, and Chaos, Physical Review A 33, 4253-4265 (1986).

[13] Wm.G. Hoover, Remark on 'Some Simple Chaotic Flows', Physical Review E 51, 759-760 (1995).

[14] J.C. Sprott, Some Simple Chaotic Flows, Physical Review E 50, R647-R650 (1994).

[15] Wm.G. Hoover and C.G. Hoover, Time-Symmetry Breaking in Hamiltonian Mechanics, arXiv 1302.2533 (2013); Computational Methods in Science and Technology 19, 77-87 (2013).

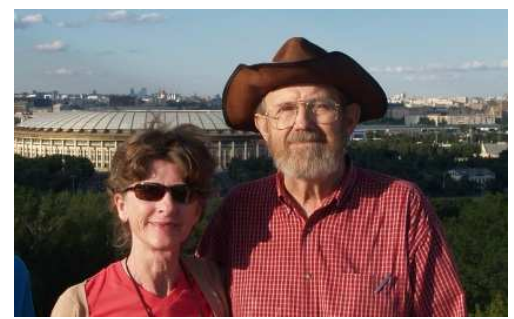

Bill and Carol Hoover met at the Lawrence Radiation Laboratory in Livermore California in 1972. Bill, with a PhD from the University of Michigan, was teaching graduate courses in numerical methods, statistical mechanics, and kinetic theory at the University of California's Livermore campus. Carol was an "A" student in plasma physics and received her PhD there in 1977. About ten years later the Hoovers got reacquainted and married before setting out on a joint Sabbatical at Keio University (Yokohama) 1989-1990. They are now retired in sparsely-settled Ruby Valley, Nevada, working on a sixth physics monograph emphasizing computational methods for controlling microscopic simulations of macroscopic behavior. 